\title{
solo \\ Primeros resultados del análisis zooarqueológico del sitio histórico El Santuario I (Magdalena, provincia de Buenos Aires)
} RMA

\author{
María Soledad García y Luis del Papa*
}

Dossier

"Facultad de Ciencias Naturales y Museo. Universidad Nacional de La Plata. CONICET. E-mail: soledad.garcia.lerena@gmail.com; loesdelpapa@hotmail.com

\begin{abstract}
Resumen
Se presentan los primeros resultados del análisis de una muestra de restos faunísticos provenientes del sitio El Santuario I (partido de Magdalena, provincia de Buenos Aires). El registro arqueológico del sitio se compone mayoritariamente de materiales vítreos pertenecientes a recipientes de bebidas alcohólicas y restos faunísticos. En menor proporción se registraron pipas de caolín y elementos metálicos. El análisis del contexto permite ubicar la ocupación en la segunda mitad del siglo XIX. A partir de las evidencias tanto documentales como arqueológicas, se interpreta al sitio como un área de consumo y descarte de grupos criollos, relacionado con las tareas pecuarias desarrolladas en el establecimiento productivo. Las evidencias de la utilización antrópica en el material óseo (marcas de corte, fracturas intencionales y señales de termoalteración) se registran exclusivamente en la fauna doméstica, Ovis aries y Bos taurus. Estas especies son las de mayor representación en la muestra y se infiere un uso diferencial de las mismas. Este trabajo permite una aproximación a la caracterización de foodways de los sectores rurales abordados y las estrategias de explotación del ganado implementadas.
\end{abstract}

Palabras clave: zooarqueología histórica; foodways; ganado; establecimiento productivo; sectores rurales.

First results of zooarchaeological analysis of El Santuario I historical site (Magdalena, Buenos Aires)

\begin{abstract}
We present the results of the analysis of faunal remains from El Santuario I (district of Magdalena, Buenos Aires). The archaeological record of the site mainly consists of glass from alcoholic beverage containers and faunal remains. Kaolin pipes and metal elements were less abundant. The analysis of the assemblage allows determining the occupation in the second half of the 19th century. From documentary and archaeological evidence, we view/ identify the site as an area of consumption and disposal related to livestock activities developed in the productive establishment of Creole groups. Evidence of human use (cut marks, intentional fractures and thermal alterations) was recorded only in domestic animals, Ovis aries and Bos taurus. These are the most representative species in the sample, [comma] evidence suggesting differential uses. This work allows characterizing feeding patterns in the rural areas analyzed and livestock management strategies.
\end{abstract}

Keywords: historical zooarchaeology; foodways; cattle; productive establishment; countryside.

La arqueología histórica en Argentina, ha reconocido un importante impulso en las investigaciones en las últimas décadas (Zarankin y Salerno 2007). Los estudios urbanos han tenido gran desarrollo, como se evidencia en la importante producción de trabajos publicados (Schávelzon 1991). Otra temática en este campo de conocimiento que ha registrado un notable desarrollo es la arqueología histórica rural, en particular los trabajos referidos a asentamientos de tipo militares en la frontera (Gómez Romero y Spota 2007) y aquellos de grupos indígenas en momentos de contacto (e.g. Pedrotta y Bagaloni 2005; Tapia y Pineau 2007). Sin embargo, la arqueología histórica rural realizada en contextos productivos criollos, no ha recibido la misma atención por parte de los investigadores, con algunas excepciones (Bagaloni 2010; Brittez 2000, 2004 y 2009; Igareta,
2002; Lanza 2006; entre otros). Este tipo de estudios posibilita el abordaje de problemáticas vinculadas a la circulación de bienes y prácticas de consumo de diversos sectores rurales, así como la articulación de diversas escalas de análisis. En este contexto, la potencialidad de los estudios en zooarqueología histórica radica en la posibilidad de abordar las prácticas cotidianas de consumo alimenticio de diferentes sectores rurales, así como interpretar el manejo del ganado en las estrategias productivas implementadas y sus cambios a través del tiempo, en un proceso amplio y complejo de conformación de la sociedad moderna.

El estudio aquí presentado, aborda el análisis del material arqueofaunístico del sitio El Santuario I, ubicado en un establecimiento productivo del partido de Magdalena, 
provincia de Buenos Aires, sobre la Ruta Provincial $\mathrm{N}^{\circ}$ 11, a $30 \mathrm{~km}$ de la ciudad homónima. El sitio se encuentra aproximadamente a $3 \mathrm{~km}$ de la costa del Río de la Plata (Figura 1), sobre un terreno elevado conformado por un cordón de conchilla de alrededor de 3-4 m de altura, con la presencia de un bosque nativo espeso de Celtis tala (tala) y Scutia buxifolia (coronillo).

A partir del análisis de la procedencia y cronología de los materiales (vítreos, cerámicos y metálicos), se sitúa al sitio arqueológico en la segunda mitad del siglo XIX. El análisis de los materiales fue articulado con fuentes documentales tales como mapas, planos catastrales, duplicados de mensura, sucesiones, periódicos y crónicas de viajeros del siglo XIX. La zona de estudio, otorgada en merced en 1636, registra una amplia historia productiva dedicada a la ganadería que continúa hasta la actualidad. A partir de las evidencias tanto documentales como escritas, se interpreta al sitio como un área de consumo y descarte de grupos criollos, posiblemente relacionado con las tareas agropecuarias desarrolladas en el establecimiento productivo (García y Paleo 2011).

El análisis del contexto arqueológico sugiere la reocupación del sitio en sucesivas oportunidades, constituyendo este lugar un punto estratégico en relación a la cercanía de los puestos, al camino de comunicación a Magdalena y Buenos Aires y a las fuentes de agua, sumado a la protección brindada por el bosque de tala. Asimismo, los indicadores socioeconómicos registrados (bebidas económicamente accesibles para la época, pipas de caolín, botones de pasta de vidrio), señalan a los trabajadores rurales como posibles generadores del contexto. Este sitio se ubica en un establecimiento mayor, que es concebido como unidad productiva en tanto conjunto de instalaciones con relaciones espaciales y materiales específicas que pueden ser abordadas arqueológicamente.

En épocas coloniales tempranas, la zona de estudio

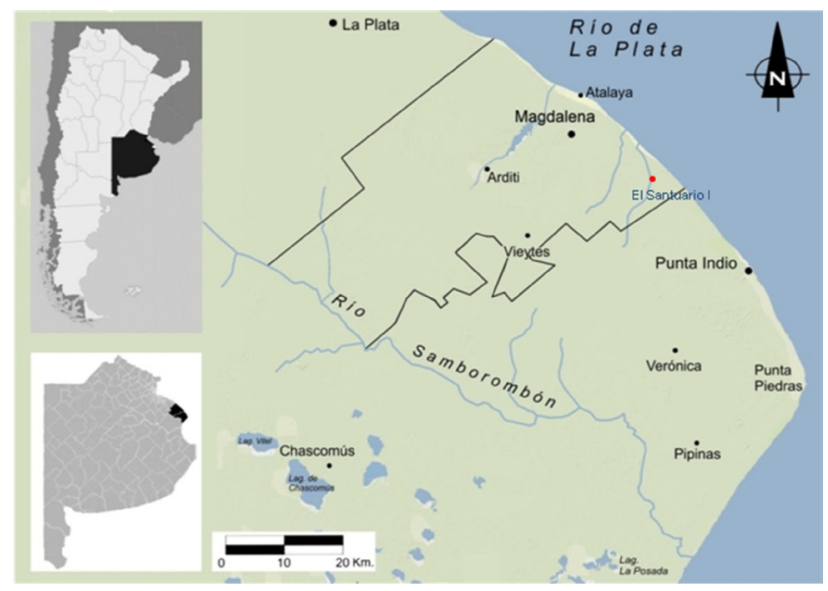

Figura 1: Ubicación del sitio El Santuario I.

Figure 1. Location of the El Santuario I. (en la frontera sur de la campaña de Buenos Aires), se caracterizó por el aprovechamiento del ganado cimarrón por sus cueros. Posteriormente la cría de ganado vacuno se convierte en una práctica de importancia, destinándose los cueros como producto de exportación y la carne para el abastecimiento de los mercados locales. Para mediados del siglo XVIII se encuentra también en los inventarios de las estancias de la campaña la presencia de ovinos, que es interpretada como un producto para el consumo de peones y esclavos, para el aprovechamiento doméstico de la lana y para el abastecimiento de la ciudad de Buenos Aires (Garavaglia 1993). Durante el siglo XIX se reconoce un cambio paulatino en la producción ganadera, en donde comienza a tomar preeminencia el ganado lanar en la campaña cercana a la ciudad, que crecerá a lo largo del siglo por la demanda en ascenso de la lana en los mercados internacionales. Mac Cann en su viaje en la década de 1840, sostiene que "(...) mi camino había transcurrido entre establecimientos dedicados a la cría de ovejas: en realidad, toda la campiña, saliendo de la ciudad y en un radio de treinta leguas, es un vasto criadero de ovejas" (Mac Cann 1969 [1853]: 60). Hilda Sábato sostiene que para las décadas 1850 y 1860 este fenómeno se configura como una verdadera "fiebre" (Sábato 1989). De esta forma, en la segunda mitad del siglo XIX, se consolida esta producción, con la introducción de razas y variedades de mejor calidad, aumentando la demanda de lanas en los mercados internacionales (Barsky y Djenderedjian 2003). En forma relacionada hay un aumento en la disponibilidad en los mercados locales de productos manufacturados europeos, que serán demandados por distintos sectores de consumo.

Así, el objetivo de este trabajo es presentar los primeros resultados del análisis zooarqueológico de una muestra de restos faunísticos provenientes del sitio El Santuario I, con el fin de caracterizar foodways de los sectores rurales en la segunda mitad del siglo XIX, e inferir estrategias de explotación del ganado.

\section{Aspectos teórico-metodológicos}

El concepto de foodways, puede definirse como un sistema de conceptualización (Brittez 2009; Landon 1996; Marschoff 2007), que involucra representaciones y dimensiones de la vida social, tanto biológicas, como económicas y culturales que hacen a la alimentación un fenómeno complejo en tanto práctica social (Marschoff 2007). De esta forma, todo acto de alimentación implica una serie de decisiones que no están guiadas únicamente por la racionalidad del costo-beneficio (Marschoff 2007; Milner y Miracle 2002). En este sentido, foodways incluirá diversos aspectos vinculados, como el acceso, distribución (a través del mercado o intercambio), preparación (faenamiento, cocción por hervido, asado, entre otros), almacenamiento, presentación y servido, consumo y descarte (Brittez 2009; Fernández 2010; 
Landon 1996; Marschoff 2007; Milner y Miracle 2002).

Consideramos que para identificar foodways de manera integral, el análisis zooarqueológico es un aspecto que debe articularse con el estudio de otras evidencias materiales, que se encuentran vinculadas a las diferentes etapas de la alimentación, así como su combinación con el aporte de fuentes documentales. Por ello, en esta primera instancia se focaliza en el análisis zooarqueológico, para abordar centralmente el componente alimenticio animal, pero se inserta e interpreta en una concepción más amplia de alimentación en tanto práctica social, poniéndose en relación con las demás evidencias del registro arqueológico y diversas fuentes documentales.

En cuanto a la información histórica se han relevado las sucesiones de la estancia desde fines del siglo XVIII hasta fines del siglo XIX y duplicados de mensura, con el fin de obtener información acerca de las actividades productivas de la misma. Por otro lado, se abordaron crónicas de viajeros (Mac Cann 1969 [1853]), fuentes literarias (Martín Fierro, de José Hernández 2000 [1872], Allá lejos y hace tiempo de G. E. Hudson 2001 [1931]) y avisos clasificados de diarios de la época para la campaña bonaerense, con el fin de obtener menciones acerca de la alimentación de sectores rurales durante mediados y fines del siglo XIX. Se concentró la atención en los tipos de alimentos, las partes seleccionadas y las formas de preparación y cocción de los mismos. Ambos corpus de información (material y documental) tienen distinta naturaleza, escala y resolución, así como la información que brindan y su rol en el seno de las prácticas sociales difiere por las condiciones mismas de su producción y utilización (Buscaglia 2010). En este trabajo se utilizan estas líneas de evidencias en forma combinada, que se refieren entre sí mutuamente y que servirán para interpretar y explicar de forma integrada el registro material e histórico.

\section{El sitio el Santuario I}

Las excavaciones se realizaron por cuadrículas de $2 \times 2$ m (Figura 2), mediante una excavación sistemática con niveles artificiales de $5 \mathrm{~cm}$, hasta una profundidad de aproximadamente $40-45 \mathrm{~cm}$, en donde se encuentra el cordón de conchillas, que constituye un nivel estéril. Hasta el momento se han excavado un total de $19 \mathrm{~m}^{2}$, y $3 \mathrm{~m}^{2}$ de sondeos. El material arqueológico se encuentra en su gran mayoría en los niveles 1 y 2, concentrándose entre los 7 y los $20 \mathrm{~cm}$ de profundidad aproximadamente.

Los materiales recuperados conforman un total de 9210 restos arqueológicos (vítreos, óseos, cerámicos y metálicos), entre los cuales 4595 corresponden a restos óseos. Para este trabajo se analiza una muestra de 1923 especímenes óseos procedentes de las dos primeras campañas de excavación. Se reconoce una gran concentración de restos arqueofaunísticos en la cuadrícula A1 y en menor medida B1 y A2 (Figura 2), y

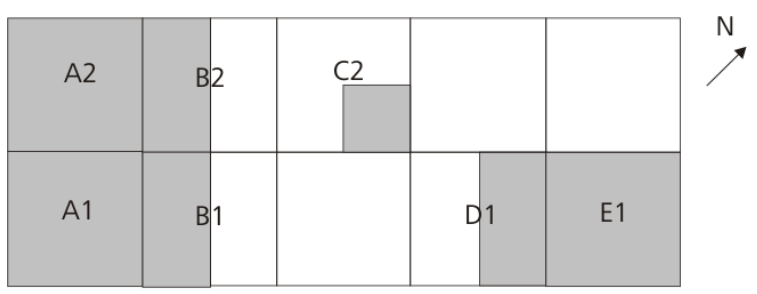

Figura 2: Planta del sitio El Santuario I. En gris, la superficie excavada.

Figure 2. Floor of the El Santuario I site. The gray area indicates the excavated surface.

son comparativamente escasos en el resto del sitio. De esta forma, el material óseo se presenta en las cuadrículas del SO del sitio, y es significativamente menor en las del sector NE. Cabe remarcar, que la cuadrícula A1 y el sector SE de la B1, tienen una densidad muy alta de materiales tanto óseos como vítreos, y presentan un sedimento con gran cantidad de ceniza color gris. Parte de este material óseo presenta señales de exposición al fuego y se ha recuperado gran cantidad de carbón vegetal de tamaño pequeño. El sector podría ser interpretado como un área de fogón a cielo abierto, posiblemente reutilizada.

\section{Materiales y métodos del análisis zooarqueológico}

Los materiales arqueofaunísticos fueron determinados taxonómicamente, en este sentido, se ha llegado a distintos niveles jerárquicos debido al grado de fragmentación de los especímenes óseos, es decir, la presencia en la muestra de una representación diferencial de rasgos anatómicos utilizados como diagnósticos para su identificación. En la categoría Mammalia se ha diferenciado en mamíferos medianos (entre $10 \mathrm{~kg}$ y $50 \mathrm{~kg}$, en este caso representados por Ovis aries y Ozotoceros bezoarticus) y mamíferos grandes ( $>$ a 50 kg; e.g. Bos taurus).

Para la cuantificación se tuvieron en cuenta los cálculos referentes a la abundancia taxonómica de la muestra en forma de NISP y MNI, este último según criterios de lateralidad y edad (Mengoni Goñalons 1999). En cuanto a la representación de partes esqueletarias se realizaron los cálculos de MNE y MAU\% (Binford 1984; Mengoni Goñalons 1999).

La preservación de los materiales ha sido analizada teniendo en cuenta el grado de meteorización de los mismos para mamíferos mayores a los $5 \mathrm{~kg}$, siguiendo los estadios propuestos por Behrensmeyer (1978). La diferencia de la densidad mineral ósea en los diferentes elementos del esqueleto puede favorecer la destrucción de algunos o parte de ellos y la supervivencia de otros (Lyman 1984). Por ello se correlacionó, a través del coeficiente de Spearman, la representación de partes esqueletarias (expresada en MAU\%) y la densidad mineral ósea (DMO) propuestas para los taxones representados (O. aries, Symmons 2004) o de una estrecha relación 
taxonómica (para B. taurus se utilizaron los valores del bóvido Connochaetes taurinus, Lam et al. 1999).

Entre las modificaciones de la superficie ósea se han tenido en cuenta aquellas producidas por agentes naturales como carnívoros, roedores, raíces y depositaciones químicas (e.g. Binford 1981; Blumenschine et al. 1996; Lyman 1994). Por otra parte, se analizaron aquellas marcas de origen antrópico como las de corte, machacado, fractura intencional, marcas de percusión y termoalteración (Binford 1981; Blumenschine et al. 1996; Mengoni Goñalons 1999). Estas modificaciones fueron analizadas a nivel macroscópico y con lupa de mano de $15 X$.

Entre las variables consideradas en el análisis de las fracturas se evaluó el estado del material óseo al momento de fracturación (fresco, seco y estadios intermedios) a través del índice de fractura fresca o FFI (Fracture Freshness Index) de Outram (2002). Para este trabajo se consideran fracturas en estado fresco (valores $0,1$ y 2 del $F F I)$, fracturas en estado intermedio, donde se produjo una cierta deshidratación del hueso o perdida de elasticidad previa a la fractura (valores 3 y 4) y fracturas en estado seco, donde el hueso perdió la mayoría o toda su elasticidad debido principalmente a factores diagenéticos o por procesos extremos (valores 5 y 6). A su vez, las fracturas se clasificaron morfológicamente en helicoidal, helicoidal/transversal, transversal, heilcoidal/ longitudinal y en forma de " $v$ ".

La determinación de la termoalteración, así como su grado de incidencia y estado del elemento cuando se produjo la acción de este agente, se realizó en base a criterios obtenidos en trabajos experimentales (Cain 2005; Merlo 2006; Shipman et al. 1984; Stiner et al. 1995). Se utilizaron como criterios principales el color, textura y uniformidad de estos atributos. En este trabajo se diferencian tres categorías básicas de termoalteración: 1- huesos quemados: son aquellos que presentan un bajo grado de termoalteración, de color marrón oscuro a negro y que por lo general se presentan en una porción del elemento; 2- huesos carbonizados: presentan un grado mayor de alteración caracterizada por elementos de color negro que generalmente se presenta de manera homogénea en todo el elemento y puede observarse una textura craquelada en su superficie; 3- huesos calcinados: de color gris a blanco, se presentan superficies craqueladas en mayor medida y la alteración compromete a la totalidad del elemento, constituyendo así en el mayor grado de alteración.

Para analizar la estructura de edad de la muestra de 0 . aries se consideraron los estadios de fusión de epífisis propuestos por Zeder (2006), en el caso de B. taurus se siguieron los criterios de Silver (1969). En este sentido, se agruparon las edades en tres categorías, crías (0 a 12 meses de edad), juveniles-subadultos (12 a 30 meses de edad) y adultos (más de 30 meses de edad). Estas categorías se representan de manera porcentual siguiendo a Kaufmann (2009). En el caso de la determinación de la edad por la erupción y desgaste dentario a partir de las mandíbulas, sólo se realizó en $O$. aries (siguiendo a Zeder 2006) ya que no se recuperaron dientes ni restos craneanos de $B$. taurus.

\section{Resultados}

De los 1923 especímenes óseos, 805 fueron asignados a alguna categoría taxonómica, que representa un $41,83 \%$ de la muestra. Cabe remarcar que la gran mayoría no identificada corresponde a especímenes menores a $2 \mathrm{~cm}$, por lo que su determinación en alguna categoría anatómica y taxonómica resulta dificultosa. Los taxones identificados se presentan en la Tabla 1. Las especies domésticas y sus taxones inclusivos componen la gran mayoría de los especímenes identificados, siendo las especies silvestres escasamente representadas.

A nivel específico, encontramos un predominio de la especie $O$. aries en la muestra, tanto a través del NISP como del MNI. En segundo lugar encontramos a $B$. taurus, con un MNI de tres individuos. Por su parte, las especies silvestres se encuentran escasamente representadas, identificandose a nivel de clase (Aves), infraclase (Teleostei), superorden (Ungulata), orden (Passeriformes), familia (Dasypodidae) y especie (Rhea americana, O. bezoarticus y Myocastor coypus).

A partir del perfil de meteorización de la muestra, se observa que un $98,55 \%$ de la misma se ubica en el estadio 1 (Behrensmeyer 1978). El 1,45\% restante se ubica en el estadio 2. Esto indica una buena preservación de la muestra, que estaría dada por un enterramiento relativamente rápido de los materiales óseos.

En cuanto a las modificaciones en la superficie ósea de origen natural, se ha identificado que el $2,66 \%$ del total

\begin{tabular}{llcc}
\hline Taxón & Nombre común & NISP & MNI \\
\hline Teleostei & & 4 & 1 \\
Aves* & Nandú & 8 & 1 \\
Rhea americana* & 14 & 1 \\
Passeriformes & 1 & 1 \\
Mammalia indeterminado & & 14 & \\
Dasypodidae & Armadillos & 1 & 1 \\
Mammalia grande & & 117 & \\
Bos taurus & Vaca & 30 & 3 \\
Mammalia mediano & & 379 & \\
Ungulata & & 50 & \\
Ovis aries & Oveja & 179 & 5 \\
Ozotoceros bezoarticus & Venado de las pampas & 5 & 1 \\
Myocastor coypus & Coipo & 2 & 1 \\
Total & & 805 & \\
\hline
\end{tabular}

Tabla 1: Abundancia taxonómica en El Santuario I. * Cáscaras de huevo.

Table 1. Taxonomic abundance of El Santuario I. * Egg shells. 


\begin{tabular}{lcc}
\hline Parte esqueletaria & Desarticulación & Descarne \\
\hline Costilla, cuerpo & 2 & - \\
Vértebra lumbar, apof. trans. & - & 1 \\
Pelvis, acetábulo & 3 & - \\
Pelvis, ilion & 1 & - \\
Pelvis, sínfisis & 1 & - \\
Fémur, epífisis proximal & 1 & - \\
Fémur, diáfisis & - & 1 \\
Fémur, epífisis distal & 1 & - \\
Tibia, epífisis proximal & 2 & - \\
Tibia, diáfisis & - & 1 \\
TOTAL & 11 & 3 \\
\hline
\end{tabular}

Tabla 2: Marcas de corte y actividades inferidas en Bos taurus y mamíferos grandes.

Table 2. Cut marks and inferred activities in Bos taurus and largesized mammals.

presenta marcas de raíces. Por su parte, se han registrado marcas de roedores en 1,35\% de los especímenes de la muestra. La mayor frecuencia de este tipo de marcas se concentra en las pelvis y costillas. Las pátinas por depositación química tienen una presencia muy baja en forma de manchas, con sólo cinco casos registrados (dos de óxido de hierro y tres de óxido de manganeso). Las marcas de carnívoros se registran en un 0,86\% de los especímenes las cuales se identificaron como pittings $(31,25 \%)$, punctures $(62,5 \%)$ y una combinación de ambos $(6,25 \%)$. Las marcas de carnívoros se registran en restos identificado de $O$. aries y $B$. taurus, así como las categorías inclusivas mamíferos grandes y medianos. Es de destacar, que en algunos casos encontramos en el mismo elemento marcas de carnívoros y marcas de procesamiento antrópico (en tres casos marcas de corte y uno con una fractura fresca con negativo de lascado).

Las marcas de origen antrópico se registran sólo en las especies domésticas $B$. taurus y $O$. aries y en las categorías abarcativas que las incluyen. Se analizan a continuación las marcas de corte, las fracturas y la termoalteración registradas en la muestra.

\section{Marcas de corte}

En la muestra no se han identificado marcas de sierra manual ni eléctrica y probablemente hayan sido producidas por filo metálico, como cuchillos. Se registra un 33\% de los especímenes de $B$. taurus y un 2,56\% de mamíferos grandes con marcas de corte. En la Tabla 2 se presentan las partes esqueletarias con marcas de corte infiriendo las actividades que las produjeron a partir de su ubicación y atributos, clasificadas en desarticulación y descarne.

Por su parte, el 31,08\% de los especímenes asignados a 0 . aries (descontando los dientes de este taxón) presenta marcas de corte, así como en la categoría mamíferos medianos se registró un 3,69\% de especímenes con este tipo de marcas. En ambas categorías las actividades inferidas fueron clasificadas en desarticulación, descarne,

\begin{tabular}{|c|c|c|c|c|c|}
\hline Parte esqueletaria & 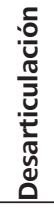 & 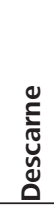 & 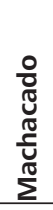 & 인 & $\begin{array}{l}\stackrel{+}{0} \\
\text { 으 } \\
\end{array}$ \\
\hline Atlas & 1 & - & - & - & - \\
\hline Vértebras cervicales & 2 & - & - & - & - \\
\hline Vértebras dorsales & 1 & - & - & - & - \\
\hline Vértebras lumbares & 3 & 6 & - & - & - \\
\hline Costillas & 6 & 2 & - & - & - \\
\hline Escápula & 1 & - & - & - & - \\
\hline Húmero, epífisis proximal & 3 & - & - & - & - \\
\hline Húmero, diáfisis & - & 3 & - & - & - \\
\hline Cúbito, epífisis proximal & 4 & - & - & - & 1 \\
\hline Radio, epífisis proximal & 1 & - & - & - & - \\
\hline Radio, diáfisis & - & 2 & - & - & - \\
\hline Radio, epífisis distal & 1 & - & - & - & - \\
\hline Pelvis, acetábulo & 2 & - & - & - & - \\
\hline Pelvis, ilion & - & 4 & - & - & - \\
\hline Fémur, epífisis proximal & 11 & - & - & - & - \\
\hline Fémur, diáfisis & - & 3 & 2 & - & - \\
\hline Fémur, epífisis distal & 1 & - & - & - & - \\
\hline Tibia, epífisis proximal & 4 & - & - & - & - \\
\hline Tibia, diáfisis & - & 1 & 2 & - & - \\
\hline $2^{\circ}$ tarsal & - & - & - & 1 & - \\
\hline Hueso plano, fragmento & 1 & - & - & - & - \\
\hline TOTAL & 42 & 21 & 4 & 1 & 1 \\
\hline
\end{tabular}

Tabla 3: Marcas de corte y actividades inferidas en Ovis aries y mamíferos medianos.

Table 3. Cut marks and inferred activities in Ovis aries and mediumsized mammals.

machacado, cuereo e indeterminado (Tabla 3).

\section{Fracturas}

Se han registrado en $B$. taurus, $O$. aries y las categorías abarcativas que las incluyen un total de 42 especímenes fracturados, de las cuales 30 se clasifican como frescas, 10 como intermedias y dos como secas. De acuerdo a sus características, se clasifican a su vez en helicoidales (55\%), helicoidal/transversal (36\%), helicoidal/ longitudinal (2\%), transversal (5\%) y en " $v$ " (2\%).

Para B. taurus, se reconocen 17 huesos largos de los cuales un $64,7 \%$ se encuentra fracturado. Estas fracturas se produjeron en estado fresco o intermedio. Uno de los fémures con fractura fresca de tipo helicoidal presenta un negativo de lascado. Los elementos en donde se registran las fracturas son el fémur (80\%), húmero y tibia (10\% cada uno). En la categoría mamiferos grandes, encontramos tres fracturas frescas en fragmentos de huesos largos, uno de los cuales presenta asociado un negativo de lascado.

En el caso de 0 . aries, de los 50 huesos largos presentes 
en la muestra se registran 26 con fracturas (52\%). Estas fracturas son clasificadas en frescas en 17 casos $(65,4 \%)$, intermedias en siete $(26,9 \%)$ y secas en dos casos $(7,7 \%)$. Los elementos anatómicos donde se registran las fracturas son el fémur en alto porcentaje, seguido por la tibia, el húmero y el radio. El miembro posterior presenta una frecuencia mayor de fracturas $(69,2 \%)$ que el anterior (30,8\%). Se encuentran asociados a las fracturas negativos de lascado en cuatro especímenes, y en un caso un hoyo de percusión. Es de destacar que en el $61,5 \%$ de los elementos de este taxón existe una asociación entre la presencia de fracturas y marcas de corte. En la categoría mamiferos medianos, se registran dos fracturas sobre diáfisis del fémur, una de ellas fresca, asociada a una marca de machacado y una fractura transversal, intermedia según el FFI.

\section{Termoalteración}

El $21 \%$ de la muestra presenta señales de exposición al fuego. De este conjunto, el $15 \%$ se encuentra parcial o totalmente quemado, el $27 \%$ carbonizado y el $58 \%$ calcinado. De esta forma, la mayoría de los restos termoalterados presenta un grado de alteración alto. Una epífisis distal de húmero y una escápula asignadas a mamíferos medianos podrían estar representando la cocción por asado, por presentar quemado un extremo del espécimen (porción que pudo quedar expuesta en momentos de la cocción). Se reconoce un 9\% para la categoría mamíferos grandes, 2,8\% para mamíferos medianos y $2,8 \%$ para mamíferos indeterminados. Por último, un 85,4\% resulta indeterminado; cabe remarcar que estos especímenes son de tamaños muy pequeños, menores a $1 \mathrm{~cm}$ de largo.

\section{Representación de partes esqueletarias}

En $B$. taurus se observa principalmente la representación del miembro posterior (Figura 3), mientras que para 0 . aries se encuentra representado la mayoría del esqueleto (Figura 4). La ausencia de costillas asignadas a estas especies se debería a la fragmentación, siendo computadas en categorías más abarcativas (e.g. mamíferos grandes y mamíferos medianos).

La representación diferencial de partes esqueletarias

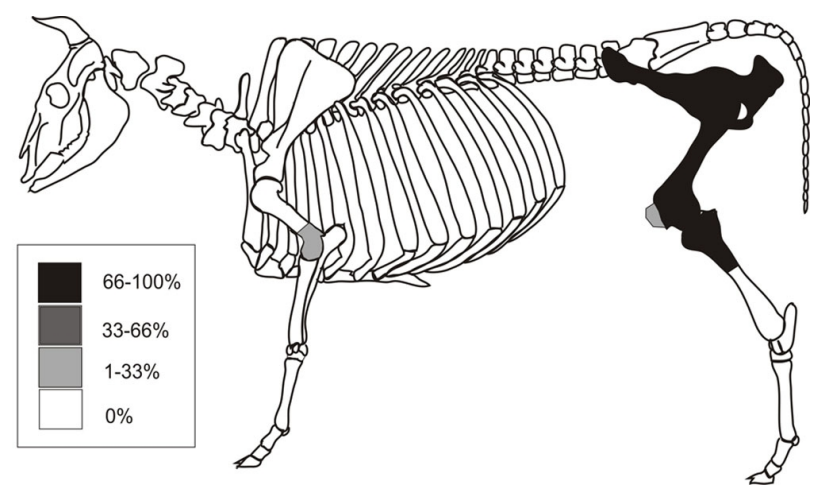

Figura 3: MAU\% de Bos taurus

Figure 3. \%MAU of Bos taurus puede deberse a procesos de preservación, por ello se realizó una correlación entre la DMO y el MAU\%, observándose valores de correlación bajos, negativos y no significativos. En el caso de $O$. aries, el valor obtenido es $r_{s}=-0,027(p>0.05, N=21)$ y para $B$. taurus es $r_{s}=$ $-0,166(p>0.05, N=57)$. De esta forma, se desprende que la representación de partes no se debe a una destrucción diferencial mediada por esta variable.

\section{Estructura de edad}

A partir del análisis de la fusión epifisaria de los elementos de $O$. aries se obtuvo la estructura de edad representada de acuerdo a los criterios previamente explicitados. Se asignó el 9,1\% de las epífisis a crías -menor a los 12 meses, con epífisis de fusión temprana sin fusionar-, $87,95 \%$ a juveniles/subadultos -con las epífisis de fusión tardía sin fusionar- y el 2,95\% restante a adultos -por las epífisis de fusión tardía que se encuentran fusionadas(Figura 5).

A partir del análisis de las mandíbulas, se asignó un individuo a la categoría crías (entre 2 y 6 meses de edad) -20\%-; uno a cría-juveniles (entre 6 y 18 meses de edad) $-20 \%$ - y tres individuos a juveniles-subadultos (dos entre 12 y 24 meses y uno de 18 meses de edad) $-60 \%$ restante. En este sentido, la fusión de los centros de osificación de los huesos largos y la erupción y desgaste dentario presentan resultados concordantes, con una preponderancia de individuos juveniles-subadultos.

Por su parte, para B. taurus, no se encuentran elementos de fusión temprana sin fusionar, por lo tanto no se encuentran crías representadas. El 80\% corresponde a elementos de juveniles/subadultos y un $20 \%$ a adultos ya que encontramos ocho elementos de fusión tardía sin fusionar y dos fusionados.

\section{Discusión}

Considerando la totalidad del conjunto analizado, según los datos que aportan el NISP y el MNI, los taxones identificados a nivel especie más representados son $O$. aries y $B$. taurus. A partir del análisis de los restos de estos animales presentes en el sitio se infiere que han sido

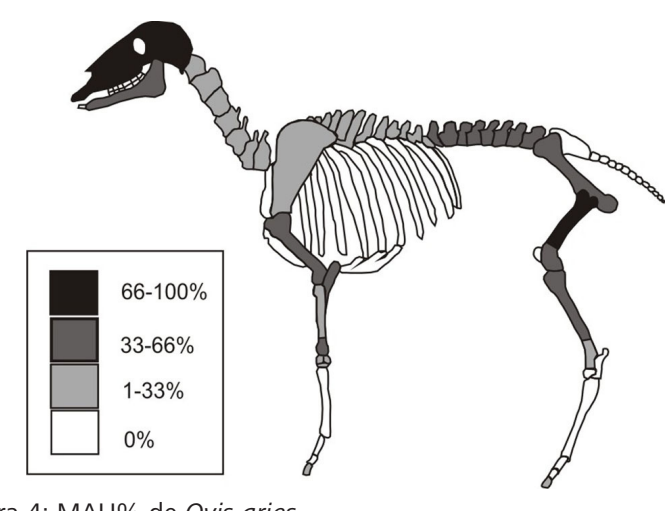

Figura 4: MAU\% de Ovis aries

Figure 4. \%MAU of Ovis aries 


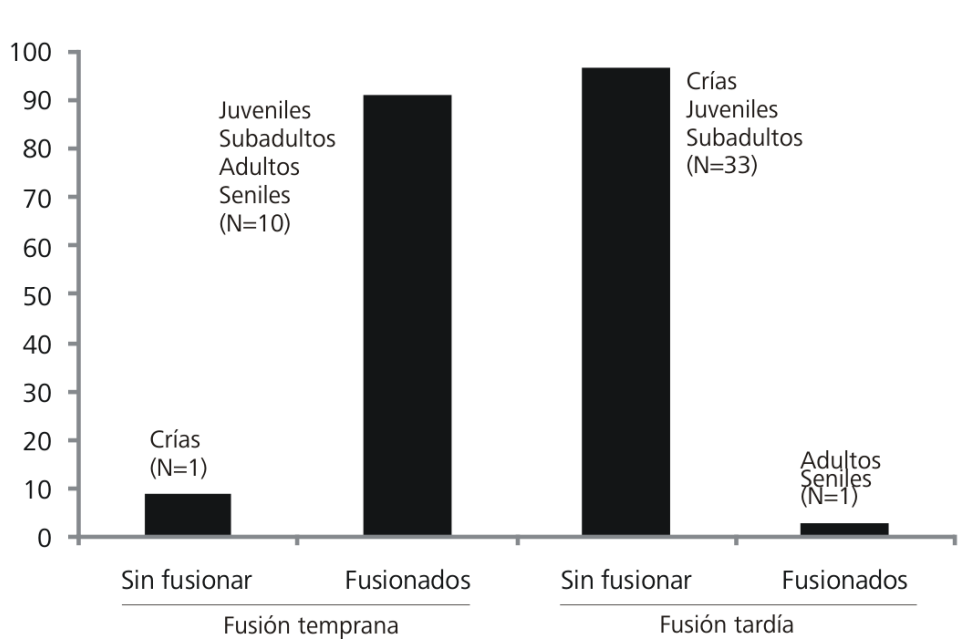

Figura 5. Elementos de Ovis aries agrupados por grupos de fusión. Fusión temprana (0 a 12 meses de edad); Fusión tardía (más de 30 meses de edad).

Figure 5. Ovis aries elements grouped for fusion categories. Early fusion (0 to 12 months of age); late fusion (more than 30 months of age).

utilizados como recurso alimenticio, contando con altas frecuencias de marcas de origen antrópico. Asimismo, la dieta posiblemente haya sido complementada minoritariamente con el consumo de fauna silvestre, como Dasypodidae, M. coypus y O. bezoarticus. Esta última, si bien no habita en la actualidad en la zona de estudio, ha sido documentada para el lapso en que fue ocupado el sitio (Pérez Meroni y Paleo 1996; Politis et al. 2011). Si bien, aun resta definir el rol que tuvieron las especies silvestres en foodways rurales por ausencia de evidencias claras, es posible que algunos de estos taxones pudieran ingresar al sitio por la utilización de su piel o cuero (Brittez 2009). La zona también presenta una amplia variedad de recursos ictícolas, tanto en el Río de la Plata como en tributarios menores, que serían accesibles a los pobladores locales, aunque los restos de peces son escasos en el registro arqueológico.

Las cáscaras de huevos asignadas a aves, por sus características podrían corresponder a domésticas en tanto que las cáscaras de huevos de Rhea americana, indicarían la recolección de silvestres. En sus memorias, Guillermo Enrique Hudson relata su consumo:

"Los huevos abundaban. Los había de gallina, de pato, de ganso y de aves silvestres. También probábamos los de pato salvaje y chorlo en la respectiva estación. En primavera -de agosto a octubre - teníamos de cuando en cuando un huevo de avestruz o rhea para la hora del desayuno" (Hudson 2001 [1931]: 192-193).

El consumo de especies silvestres por los pobladores urbanos durante el siglo XIX, está ampliamente documentado para la época. Perdices y pequeños mamíferos se ofrecían en el mercado de Buenos Aires. Por su parte, en su viaje por las pampas argentinas, William Mac Cann relata en más de una ocasión la práctica de caza de animales silvestres: "Nos entretuvimos cazando armadillos, que constituyen un buen manjar. Las perdices son tan abundantes y mansas que las matábamos con los rebenques: de esta suerte nos procuramos un excelente almuerzo." (Mac Cann 1969 [1853]: 69). Si bien las menciones en sitios fortificados son abundantes, las fuentes que refieren al consumo de fauna silvestre en ámbitos rurales son escasas, correspondiéndose con la baja representación de sus restos en el sitio analizado.

En cuanto a la accesibilidad de los recursos domésticos mayoritarios, los inventarios de las testamentarías y sucesiones relevadas para la estancia, presentan algunos datos relevantes a tener en cuenta. En el primer documento realizado para esta unidad productiva, la sucesión del año 1791 de Don Januario Fernández, un importante estanciero tardocolonial, se encuentra documentada la presencia de 36 bueyes, 69 mulas, 611 ovejas, 1243 yeguas y 2424 vacas ${ }^{1}$. Casi un siglo más tarde, en la testamentaría de Don José Sixto Fernández iniciada en $1881^{2}$, esta estancia cuenta con 4 y $1 / 4$ leguas cuadradas, en la que se inventarían la edificación principal (denominada Primera Estancia) y 14 puestos de estancia (Figura 6). Es de destacar, que todos los puestos y el casco principal cuentan con corrales para ovejas, pero únicamente el casco de estancia cuenta con dos corrales para hacienda vacuna ${ }^{2}$. De esta forma, las mismas actividades productivas de la estancia brindan el acceso a los recursos domésticos. En estos dos inventarios sucesivos del mismo "establecimiento de campo", como es referido en las fuentes, podemos vislumbrar el cambio de la orientación productiva. Diversos autores, postulan que el consumo de carne de oveja se remonta a momentos previos al auge de la producción ovina de la segunda mitad del siglo XIX (Brittez 2000; Garavagia 1993; Mayo 1995). Por su parte, en el inventario realizado en 1881, si bien no cuenta con los valores totales de ganado, consigna corrales de ovejas en todos los puestos, lo que nos permite sostener que la producción mayoritaria de la estancia estaba orientada hacia la cría de ganado lanar. En su estadía en Magdalena, Mac Cann sostiene acerca de la estancia de Mr. Taylor ubicada en este pago: "Debe considerarse que se trata de terrenos inmejorables para la cría de ovejas, distantes apenas quince leguas de la ciudad de Buenos Aires" (Mac Cann 1969 [1853]: 29). En la segunda mitad del siglo XIX, en pleno auge de la fiebre lanar, "cordero y capón terminaron por invadir la mesa de los trabajadores rurales y formaron parte de la dieta cotidiana de grandes sectores de la población de Buenos Aires durante varias décadas" (Sábato 1989: 35). Los

\footnotetext{
1 AGN. Sucesión 5874.

2 AGN. Sucesión 5796.
} 


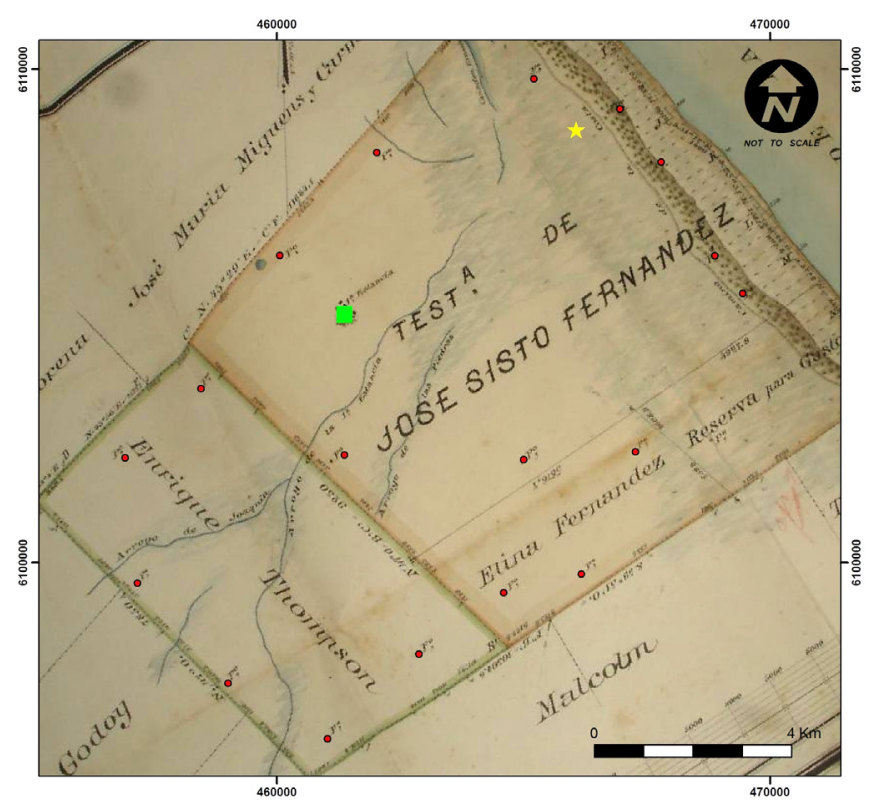

Figura 6: Duplicado de mensura de la Testamentaría de Sixto Fernández, finalizada en 1885 (Duplicado 311, Magdalena). Referencias: Circulos: puestos de estancia, Cuadrado: casco principal y Estrella: el sitio arqueológico.

Figure 6. Measurement duplicate of the Testamentaría de Sixto Fernández, finalized in 1885 (Duplicate 311, Magdalena). References: Circles: position of ranches, Square: main hull, and Star: the archaeological site.

diarios relevados ${ }^{3}$, señalan esta orientación productiva de la campaña bonaerense cercana a la ciudad para la segunda mitad del siglo XIX. En los avisos y anuncios registrados, se ofrecen ovejas y majadas enteras para su compra y reproductores de razas importados, así como productos que se relacionan con la producción de lanas: tijeras para esquilar, hilos para atar la lana y productos sarnífugos.

William Mac Cann, por su parte y desde su mirada extranjera, sostiene acerca de los paisanos de las pampas que "su alimento consiste exclusivamente en carne de vaca y de cordero" (Mac Cann 1969 [1853]:32). También Hudson relata que "El desayuno se componía de costillas de cordero, preparaciones de maíz y café" (Hudson 2001 [1931]: 192).

Teniendo en cuenta los inventarios para la época, el ganado estaba presente en el mismo establecimiento productivo, por lo que los individuos serían seleccionados de los mismos rebaños y faenados en la estancia. A partir del estudio del MAU\% observamos que está representado la mayoría del esqueleto de 0 . aries, las costillas posiblemente computadas en mamíferos medianos, por lo que su aprovechamiento sería integral. Según el rango etario identificado para esta especie, podemos inferir que predomina el aprovechamiento de estos recursos para el consumo de carne (por la mayor

\footnotetext{
3 Diarios "El Monitor de la campaña", de Exaltación de la Cruz (18711873); "Amigo del Pueblo de San Nicolás", de San Nicolás (18671868)
}

proporción de individuos juveniles/subadultos).

Para B. taurus se habría dado un aprovechamiento de los miembros posteriores y posiblemente costillas, principalmente como producto de un consumo de partes de buen rinde económico. Las partes faltantes de esta especie pueden interpretarse por el transporte a zonas de comercio de las regiones cercanas o por reparto entre puesteros dentro de la misma estancia. Debido a que sólo el casco principal contaba con corrales para ganado vacuno, además de ser el espacio de concentración de capataces, encargados y esporádicamente los dueños de la estancia, consideramos que la hipótesis más factible para explicar la representación de partes esqueletarias en el registro arqueológico es que en el casco se habrían realizado las tareas de matanza, despostamiento primario, distribución y circulación de la carne vacuna. De esta forma se centralizaría desde las esferas de poder de la estancia el manejo de este recurso, regulando el consumo de los peones y puesteros, lo que habría generado un aprovechamiento diferencial de las partes reflejadas en el MAU\% de esta especie.

En cuanto a las técnicas de faenamiento, en la muestra analizada no hay cortes de sierras manual ni eléctrica. El corte de sierra es una práctica que se realiza luego de pasada la mitad del siglo XIX. El corte de media res en particular, se implementa a principios del siglo XX y no se registra en los materiales analizados aquí. Las marcas de corte, a partir de su funcionalidad inferida, nos indican que se utilizarían elementos como cuchillos para la desarticulación de los huesos y para el descarne de los mismos. Las fracturas intencionales en los huesos largos, con negativos de lascado, hoyos de percusión y realizadas en estado fresco e intermedio, estarían indicando el consumo de médula ósea, rica en grasas.

En cuanto a la cocción de los alimentos, ésta podría estar evidenciada por la existencia de restos que presentan atributos relacionados con la alteración térmica sólo en una de las epífisis, lo que sugiere que éstos pudieron ser asados con tejidos que protegen el resto del elemento (Gifford-Gonzalez 1989; Kent 1993). Se han registrado en el conjunto algunas señales de termoalteración de este tipo, incluidos en la categoría quemados, pero son poco frecuentes. Diversas formas de cocciones eran corrientes para la época, tal como señala el poema Martín Fierro: "Venía la carne con cuero,/la sabrosa carbonada,/la mazamorra bien pisada,/los pasteles y el buen vino..." (Hernández 2000 [1872]: 20), y G. E. Hudson "Comimos asado, carne de puchero, pan y vino (...)" (Hudson 2001 [1931]: 136). Las cocciones de tipo asado eran las más frecuentes en ámbitos rurales, tal como lo documentan innumerables fragmentos de viajeros:

“Éste [el asador] era una barra de hierro de unos cuantos pies de largo. Don José le ayudó a ensartar la carne y a asegurar un extremo del asador 
clavándolo en el suelo de manera que quedara inclinado sobre las brasas. En esta forma la carne se asa muy bien, porque el calor, subiendo de todos lados, la penetra completamente dándole un sabor muy especial y delicado" (Mac Cann 1969 [1853]: 46).

De acuerdo a los análisis realizados por Mario Silveira (1999) en contextos domésticos urbanos, la escasa presencia de marcas de corte se podría relacionar con las formas de cocción. En preparados tipo guisados y pucheros, producto del hervido de larga duración, la carne se desprendería más fácilmente de los huesos por lo que serían menos frecuentes las marcas de corte y raspado. En cocciones tipo asado, serían más frecuentes las marcas de corte. La relación porcentual de la presencia de marcas de corte en el material analizado para este trabajo es alto, alrededor del $30 \%$ para $B$. taurus y 0 . aries.

Las prácticas de servido y presentación de los alimentos involucran una serie de elementos materiales, tales como recipientes de loza y vidrio, en forma de platos, bandejas y fuentes. Para abordar este aspecto de las prácticas alimenticias es necesaria su articulación con los demás elementos del registro arqueológico. El contexto del sitio El Santuario I, se compone exclusivamente de contenedores de bebidas de vidrio, material zooarqueológico, pipas de caolín y elementos metálicos (bombillas, clavos). No se han registrado elementos de loza de ningún tipo, pese a ser muy frecuentes y conspicuos en contextos del siglo XIX (Schávelzon 1991), así como tampoco se han registrado recipientes de vidrio destinados a servir (copas, vasos). De acuerdo a las fuentes consultadas, la práctica habitual en ámbitos rurales era servirse directamente del asador la porción de carne a consumir. Nuevamente Mac Cann nos ilustra al respecto:

“Entonces clavaron el fierro en el suelo, nos sentamos alrededor y empezamos a cortar con nuestros propios cuchillos, muy contentos de participar en aquel banquete de gitanos (...) Comer de esta guisa requiere cierta práctica: primeramente se ha de coger la carne con la mano izquierda, luego tomar con los dientes el bocado elegido y aplicar el cuchillo, con la mano derecha, apoyando el filo hacia arriba para cortar" (Mac Cann 1969 [1853]: 46).

Diversas secciones o partes del animal eran elegidas para su consumo. En cuanto a la oveja, era frecuente la práctica de asar el animal entero, así como los costillares. Su carne también era aprovechada en cocciones tipo guisos y estofados. El consumo de carne ovina está ampliamente documentado en sitios arqueológicos urbanos y rurales. Mario Silveira sostiene que "El testimonio arqueológico mostró un consumo superior a la expectativa que nos sugerían los testimonios escritos, en particular los del siglo XIX. También este consumo tenía un componente constante de animales juveniles (corderos) junto con adultos (carneros)" (Silveira 1999: 393). Por su parte, Fernando Brittez a partir del trabajo en la Estancia Las Vizcacheras, en el partido de Brandsen asegura que "es clara la preeminencia de Ovis aries en la dieta" (Brittez 2000: 177). En este último sitio también se registra para esta especie la totalidad del esqueleto representado. Por su parte, la carne vacuna era frecuentemente consumida por todos los sectores socioeconómicos. Era utilizada para distintos preparados como guisados, estofados y asado. Las fuentes mencionan en forma corriente los asados con cuero, en donde se asaba el animal completo, y la preferencia de ciertas partes como el matambre, que no tienen un componente óseo que pueda abordarse arqueológicamente. De acuerdo al estudio comparativo realizado por Brittez (2000, 2009) de las especies consumidas en los contextos de estancias, entre el 42 y $77 \%$ corresponden a 0 . aries y entre el 2 y $16 \%$ a ganado vacuno.

Un aspecto cultural relevante de la noción de foodways son las rutinas diarias de alimentación. De acuerdo a las fuentes consultadas, los paisanos "tienen por costumbre desayunarse con mate y en realidad lo beben todo el día. A eso de las once de la mañana comen carne y consumen el mismo alimento por la noche, una hora después de entrado el sol." (Mac Cann 1969 [1853]: 32). Asimismo, las comidas se realizaban de forma colectiva, en nuestro caso de análisis posiblemente alrededor o en las cercanías de un fogón, en combinación con otras prácticas de consumo, como el de bebidas alcohólicas, de tabaco (presencia de pipas de caolín con señales de uso), y posiblemente el consumo de una infusión como el mate (presencia de dos bombillas en el registro). "[El paisano] Cuando está ocioso, se le hallará siempre fumando o tomando mate" (Mac Cann 1969 [1853]: 117).

El descarte se produce en sucesivas etapas del proceso alimenticio, posiblemente en más de un sector. Así, los elementos desechados en un procesamiento primario no necesariamente serán descartados en la misma zona que los del consumo final (e.g. B. taurus). En el sitio El Santuario I, las pipas de caolín se descartarían luego de su rotura (no se han hallado enteras y eran muy frágiles, Schávelzon 1991). Los recipientes de bebidas alcohólicas también, ya que en el contexto analizado, no se ha hallado ningún recipiente entero (de un número mínimo calculado de 39 recipientes). Para la época era común el rellenado así como la compra en almacenes y pulperías de recipientes vacíos, por lo que no sería habitual el descarte de botellas enteras. Asimismo, presentan las mismas pátinas iridiscentes en las superficies de fractura, producto de la meteorización química, lo que indicaría que ingresaron al contexto arqueológico una vez rotas. El material óseo, por su parte, sería descartado en el fuego y sus inmediaciones. Estos a su vez, podrían ser aprovechados por carnívoros, ya que se encuentran 
superpuestas marcas de éstos y marcas antrópicas. Los carnívoros que han dejado estas marcas probablemente hayan sido perros domésticos, ya que está ampliamente documentada para la época la presencia de este animal acompañando las faenas del campo (Hudson 2001 [1931]; Mac Cann 1969 [1853]), a los que alimentarían con los desechos.

En cuanto a la termoalteración registrada, es de destacar que este tipo de atributos no suele representar el producto total de la cocción de los alimentos sino también el descarte por cuestiones de higiene, para impedir la acción de carroñeros o como combustible (Cain 2005; Costamagno et al. 2005; De Nigris 2004; Gifford-Gonzalez 1989; Kent 1993). Para este análisis se desechó la posibilidad del uso de los huesos como combustible principal, debido a la gran cantidad de madera de buena calidad que se encontraría en las inmediaciones (tala, coronillo). El descarte en lugares de combustión se podría inferir por una mayor proporción de restos donde el quemado compromete a toda la superficie de los mismos y con un alto grado de alteración. De esta actividad se espera una mayor proporción de fragmentos calcinados (De Nigris 2004; Kent 1993; Oliver 1993), tal como se registra en este sitio. Debido al alto grado de termoalteración registrado, así como por la disponibilidad de maderas potenciales como combustible, consideramos que el material óseo es el resultado del consumo alimenticio y luego habrían sido arrojados al fuego para su descarte y a su vez como combustible auxiliar, como se registra en otros casos de la región pampeana (e.g. Sitio Siempreverde, Lanza 2006).

\section{Palabras finales}

El estudio de la representación de partes esqueletarias, el estado de fragmentación y las marcas antrópicas registradas, nos permiten proponer que en el sitio El Santuario I no se encuentran cortes comerciales, lo que estaría indicando que no habría existido una compra en el mercado de las carnes consumidas, caracterizándose como un patrón de consumo rural (Silveira y Bogan 2007). Las actividades productivas ganaderas realizadas en la estancia habrían permitido un fácil acceso a las especies domésticas consumidas. Asimismo, se registra un consumo diferencial de las mismas, lo que indicaría una circulación de la carne vacuna, posiblemente distribuida desde el casco de la estancia, y un consumo integral del ovino en los puestos y sus inmediaciones.

El análisis de los materiales en forma integral así como su interpretación contextual y articulación con fuentes documentales, permite aproximarnos a conocer la interrelación de las prácticas e inferir status socioeconómico en base a las presencias, ausencias y asociaciones en el registro arqueológico. De esta forma, es posible reconstruir aspectos de la vida cotidiana de los actores sociales e interpretar foodways.
Los contenedores de bebidas alcohólicas hallados, eran productos importados de consumo masivo y económico, y fueron el resultado del proceso de industrialización de los países centrales. Este proceso es concomitante con la consolidación de la incorporación de la región pampeana en la segunda mitad del siglo XIX al mercado mundial como agroexportadora, principalmente a través de los productos vinculados al ganado lanar. Así, a partir de un análisis de un contexto particular, de la escala de sitio arqueológico, es posible abordar e interpretar las prácticas cotidianas de alimentación y consumo de un conjunto de peones y puesteros de una estancia dedicada a la producción ganadera, en donde las articulaciones de escalas nos permiten abordar la confluencia de mercancías provenientes del comercio ultramarino en un proceso de circulación en donde la producción agropecuaria de la estancia encontraba mercados locales e internacionales de inserción. Este tipo de análisis pretende aportar al conocimiento de los procesos de conformación de la sociedad moderna en el ámbito rural pampeano a partir de brindar elementos para analizar la construcción de identidades colectivas, particularmente aquellas vinculadas a sectores trabajadores.

La Plata, 29 de octubre de 2011

\section{Agradecimientos}

A nuestros directores Dr. Luciano de Santis y Lic. Clara Paleo por las sugerencias y lectura del manuscrito. A Rocío Gambaro por su ayuda con el inglés. Este trabajo forma parte de la tesis doctoral de uno de los autores, realizado en el marco de una beca doctoral de CONICET.

\section{Fuentes}

Archivo General de la Nación. Sucesiones No 5874 y 5796.

Duplicados de Mensura No 264 de 1863 y 311 de 1885. Partido de Magdalena. Archivo de Geodesia y Catastro de la Provincia de Buenos Aires. Ministerio de Obras Públicas. Diarios "El Monitor de la campaña", de Exaltación de la Cruz (1871-1873); "Amigo del Pueblo de San Nicolás", de San Nicolás (1867-1868). Hemeroteca de la Universidad Nacional de La Plata.

\section{Bibliografía}

Bagaloni, V. 2010. Desde las orillas... una comparación de los sitios La Libertad (Partido de San Cayetano) y las Toscas (Partido de Tres Arroyos), Buenos Aires. En: M. Berón, L. Luna, M. Bonomo, C. Montalvo, C. Aranda y M. Carrera Aizpitarte (eds). Mamül Mapu: pasado y presente desde la arqueología pampeana. Tomo II. 403-418. Editorial Libros del Espinillo, Ayacucho.

Barsky, O., J. Djenderedjian. 2003. Historia del Capitalismo Agrario Pampeano. Tomo 1: La expansión ganadera hasta 1895. Editorial. Siglo XXI. Buenos Aires. 
Behrensmeyer, A. F. 1978. Taphonomic and ecologic information from bone weathering. Paleobiology, 4: 150-162.

Binford, L. R. 1981. Bones: Ancient Men and Modern Myths, Academic Press, New York.

Binford, L. R. 1984. Faunal Remains from Klasies River Mouth, Academic Press, Orlando.

Blumenschine, R.J., C.W. Marean y S.D. Capaldo. 1996. Blind test of inter-analyst correspondence and accuracy in the identification of cut marks, percussion marks, and carnivore tooth marks on bone surfaces. Journal of Archaeological Science, 23: 493-507.

Brittez, F. 2000. La comida y las cosas: una visión arqueológica de la campaña bonaerense de la segunda mitad del siglo XIX. C. Mayo (ed). Vivir en la frontera: la casa, la dieta, la pulpería, la escuela (1770-1870). 169199. Editorial Biblos. Buenos Aires.

Brittez, F. 2004. Arqueología rural en el Partido de Coronel Brandsen, provincia de Buenos Aires. C. Gradín y F. Oliva (eds). La Región pampeana: su pasado arqueológico. 211-222. Laborde Editores. Rosario.

Brittez, F. 2009. Zooarqueología, tafonomía y procesos de formación de sitios rurales pampeanos: estado de la cuestión y expectativas para momentos tardíos. Revista de Arqueología Histórica Argentina y Latinoamericana 3: 47-68

Buscaglia, S. 2010. Teoría poscolonial y arqueología histórica. Aportes para una discusión crítica sobre poder, contacto y colonialismo. R. Bárcena y H. Chiavazza (eds) Arqueología Argentina en el Bicentenario de la Revolución de Mayo.755-760. Facultad de Filosofía y Letras. UNCuyo. Mendoza.

Cain, C. R. 2005. Using Burned animal bone to look at Middle Stone Age occupation and behavior. Journal of Archaeological Science, 32: 873-884.

Costamagno, S., I. Théry-Parisot, J.P. Brugal y R. Guibert. 2005. Taphonomic consequences of the use of bones as fuel: experimental data and archaeological consequences. O'Connor, T. (ed), Biosphere to Lithosphere. New Studies in Vertebrate Taphonomy, pp. 51-62. Oxbow Books, Oxford.

De Nigris, M. E. 2004. El consumo en grupos cazadores recolectores. Un ejemplo zooarqueológico de patagonia meridional. Sociedad Argentina de Antropología, Colección Tesis Doctorales, Buenos Aires.

Fernández, P. M. 2010. Cazadores y presas: 3.500 años de interacción entre seres humanos y animales en el noroeste de Chubut. Fundación de Historia Natural Félix de Azara, Buenos Aires.

Garavaglia, J.C. 1993, Las 'estancias' en la campaña de Buenos Aires. Los medios de producción. Fradkin, R. (ed). La historia agraria del Río de la Plata colonial: Ios establecimientos productivos. Vol. 11, 124-208. Centro Editor de América Latina. Buenos Aires.

García, M. S., M.C. Paleo. 2011. El sitio El Santuario I: arqueología histórica rural de la Magdalena del siglo XIX. Libro de Resúmenes del $1^{\circ}$ Congreso Internacional de Arqueología de la Cuenca del Plata. Buenos Aires.

Gifford-González, D. 1989. Ethnographic Analogues for Interpreting Modified Bones: Some Cases form East Africa. Bonnichsen, R. y M. Sorg (eds), Bone Modification, 179-246, University of Maine, Orono.

Gómez Romero, F. y J. Spota. 2007. Algunos comentarios críticos acerca de 15 años de arqueología en los fortines pampeanos. Relaciones de la Sociedad Argentina de Antropología . Tomo XXXI. 161-185.

Hernández, J. 2000 [1872]. El gaucho Martín Fierro. Editorial Sol. Barcelona.

Hudson, G.E. 2001 [1931]. Allá lejos y hace tiempo. Ediciones El Aleph. Buenos Aires.

Igareta, A. 2002. La prehistoria de la historia: Arqueología histórica en el Paseo del Bosque de La Plata. Arqueología Histórica Argentina. Actas del I Congreso Nacional de Arqueología Histórica. 723-732. Mendoza, Ed. Corregidor.

Kaufmann, C. 2009. Estructura de Edad y Sexo en Lama guanicoe (Guanaco). Estudios actualísticos y arqueológicos en Pampa y Patagonia, Sociedad Argentina de Antropología, Buenos Aires.

Kent, S. 1993. Variability in Faunal Assemblages: the Influence of Hunting Skill, Sharing, Dogs and Mode of Cooking on Faunal Remains at a Sedentary Kalahari Community. Journal of Anthropological Archaeology, 12: 323-383.

Lam, Y. M., X. Chen y O. M. Pearson. 1999. Intertaxonomic variability in patterns of bone density and differential representation of bovid, cervid, and equid elements in the archaeological record. American Antiquity, 64: 343-362.

Landon, D. B. 1996. Feeding Colonial Boston: A Zooarchaeological Study. Journal of the Society for Historical Archaeology 30 (1):1-153.

Lanza, M. 2006. Estudio zooarqueológico del sitio Siempreverde. Relaciones de la Sociedad Argentina de Antropología 31:229-247. 
Lyman, R. L. 1984. Bone Density and Differential Survivorship of Fossil Classes. Journal of Anthropological Archaeology, 3: 259-299.

Lyman, R. L. 1994. Vertebrate Taphonomy, Cambridge University Press, Cambridge.

Mac Cann, W. 1969 [1853] Viaje a caballo por las provincias argentinas. Solar/Hachette. Buenos Aires.

Marschoff, M. 2007. Gato por liebre. Prácticas alimenticias en Floridablanca. Ed. Teseo. Buenos Aires.

Mayo, C. 1995. Estancia y sociedad en la pampa. 17401820. Editorial Biblos. Buenos Aires.

Mengoni Goñalons, G. L. 1999. Cazadores de guanacos de la estepa patagónica, Sociedad Argentina Antropología, Colección tesis Doctorales. Buenos Aires.

Merlo, J. 2006. Investigaciones actualísticas experimentales para la interpretación del registro arqueofaunístico en los sitios fortificados del siglo XIX. En Funari y Brittez comp. Arqueología Histórica en América Latina: temas y discusiones recientes. Mar del Plata. Ediciones Suárez.

Milner, N. y P. Miracle. 2002. Introduction: patterning data and consuming theory. P. Miracle y N. Milner (eds), Consuming passions and patterns of consumption, pp. 1-15, McDonald Institute for Archaeological Research, University of Cambridge, Cambridge.

Oliver, J. O. 1993. Carcass Processing by the Hadza: Bone Breakage from Butchery to Consumption. Hudson, J. (ed), From bones to behavior: ethnoarchaeological and experimental contributions to the interpretation of faunal remain, pp. 200-227, Center for Archaeological Investigations, Occasional Paper 21, Southern Illinois University at Carbondale.

Outram, A. K. 2002. Bone fracture and withinbone nutrients: an experimentally based method for investigating levels of marrow extraction. Miracle, P. y N. Milner (eds), Consuming Passions and Patterns of Consumption, pp. 51-62, McDonald Institute for Archaeological Research, Cambridge.

Pedrotta, V., V. Bagaloni. 2005. Looking at Interethnic Relations in the Southern Border Through Glass Remains: The Nineteenth- Century Pampa Region, Argentina. International Journal of Historical Archaeology 9 (3): 177-193.

Pérez Meroni M. y M. C. Paleo. 1996. Don Gerardo. Un nuevo sitio arqueológico en el Partido de Punta Indio, Provincia de Buenos Aires. M. A. Caggiano (comp.) Primeras Jornadas Chivilcoyanas en Ciencias Sociales y Naturales. 187-190. Chivilcoy.
Politis, G. G., L. Prates, M.L. Merino y M.F. Tognelli. 2011. Distribution parameters of guanaco (Lama guanicoe), pampas deer (Ozotoceros bezoarticus) and marsh deer (Blastocerus dichotomus) in Central Argentina: Archaeological and paleoenvironmental implications. Journal of Archaeological Science, 38: 1405-1416.

Sábato, H. 1989. Capitalismo y Ganadería en Buenos Aires. La fiebre del lanar 1850-1890. Editorial Sudamericana. Buenos Aires.

Schávelzon, D. 1991. Arqueología Histórica en Buenos Aires. La cultura material porteña de los siglos XVIII y XIX. Editorial Corregidor. Buenos Aires.

Shipman, P, G. F. Foster y M. Schoeninger. 1984. Burnt bones and teeth: an experimental study of colour, morphology, crystal structure and shrinkage. Journal of Archaeological Science, 11: 307-325.

Silveira, M. 1999. Zooarqueología Histórica Urbana: Ciudad de Buenos Aires. Tesis Doctoral, Facultad de Filosofía y Letras, Universidad de Buenos Aires, Buenos Aires, Argentina, 442 pp.

Silveira M., S. Bogan. 2007. Valoraciones en Zooarqueología Histórica. E. Néspolo, M. Ramos y G. Goldwaser (comp). Signos en el tiempo y rastros en la tierra. Volumen II. 167-174. Univ. Naci. de Luján. Luján.

Silver, I. 1969. The ageing of domestic animals. D. Brothwell y E. Higgs (eds). Science in Archaeology, (2 ${ }^{\text {nd }}$ edition), 283-302. Thames. London.

Stiner, M. C., S. L. Kuhn, S. Weiner y O. Bar-Yosef. 1995. Differential burning, recrystalization, and fragmentation of archaeological bone. Journal of Archaeological Science, 22: 223-237.

Symmons, R. 2004. Digital photodensitometry: a reliable and accessible method for measuring bone density. Journal of Archaeological Science, 31: 711-719.

Tapia, A., V. Pineau. 2007. Precisando la cronología de un sitio ranquel a través de los fragmentos vítreos. F. Oliva, N. de Grandis y J. Rodriguez comp. Arqueología Argentina en los inicios de un nuevo siglo. Tomo 1. 473484. Laborde editor. Rosario.

Zarankin, A., M. Salerno. 2007. El sur por el sur. Una revisión de sobre la historia y el desarrollo de la Arqueología Histórica en América Meridional. Vestigios $1(1): 17-48$.

Zeder, M. A.2006. Reconciling Rates of Long Bone Fusion and Tooth Eruption and Wear in Sheep (Ovis) and Goat (Capra). Deborah Ruscillo (ed). Recent Advances in Ageing and Sexing Animal Bones, 87-118. Oxbow Books. Oxford. 